\title{
Ueber die chirurgische Behandlung der Otitis media chronica sicca.
}

(Vortrag, gehalten am XII. internationalen medicinischen Congress zu Moskau.)

Von

Dr. E. J. Moure (Bordeaux).

Die meisten Behandlungsmethoden der Otitis media chronica sicea haben sich bisher als völlig unzureichend erwiesen, insofern durch keine derselben eine Besserung, geschweige eine Heilung dieses Krankheitszustandes erzielt werden konnte. Auf Grund dieser Erfahrungen hat man sich der chirurgischen Behandlung zugewendet, zumal diese, bei Beachtung der Regeln der Asepsis und Antisepsis, gegenwärtig keine weiteren gefährlichen Folgen für das Gehörorgan und den Allgemeinzustand mit sich bringt, ein bedeutender Fortsehritt gegentiber einer früheren Zeit, wo sohon eine einfache Paracentese manchmal von böchst bedrohlichen Allgemeinerscheinungen gefolgt war, wie ich dies selbst in meiner Praxis zu beobachten Gelegenheit hatte.

Von den Otologen aller Länder wurden im Laufe der Zeit die versehiedensten Operationsmethoden, einfacher und complicirter Art, versuchsweise in Anwendung gebracht, um die progressive Taubheit, welehe in den meisten Fällen sich als eine Folge der Otitis media ohronica sicea darstellt, mit Erfolg zu behandeln.

Wir sind nicht der Ansicht, dass allem, was seit 15 Jahren diesbeztiglich vorgeschlagen wurde, ein ansehnlicher praktischer Werth zukomme. Ein grosser Theil daron bietet lediglich historisches Interesse und kann daher hier tibergangen werden.

Die von W eber-Liel in die Praxis eingefibhrte Tenotomie fand an einzelnen Otologen, unter anderen an dem Franzosen Miot eifrige Anhänger. Von letzterem rührt eine Arbeit her, 
die über alle technischen Details der Operation wie auch uber die von ihr zu erwartenden Resultate Aufsehluss giebt. Miot hat als einer der ersten die operative Therapie der in Rede stehenden Affection in Frankreieh eingeführt und hält auch jetzt noob mit grosser Energie an seinen Ideen fest, indem er neben der Tenotomie und der Myringodektomie auch noch die Mobilisation des Stapes und in letzter Zeit auch noch die Extraction der Gehörknöchelehen ausführt. In mehreren Publicationen behandelt er Indication und Contraindication dieser Methoden, welche weiter unten noch Erwähnung finden sollen.

Ehe wir auf das eigentliche Thema unseres Vortrages ubergehen, wollen wir nur noch bemerken, dass wir uns anssehliesslich mit der genuinen Otitis media chronica sicea beschäftigen wollen und alle anderen adhäsiven Processe, wie sie als Folgen von Eiterungen und Exsudationen im Mittelohr vorkommen, nicht berticksichtigen.

\section{Indicationen der Operation.}

In seiner Arbeit über die Tenotomie und Mobilisation des Stapes macht Kessel ${ }^{1}$ ) mit Recht darauf aufmerksam, dass bei der Mittelohrsklerose nicht nur eine Erstarrung des schalleitenden Apparates sich findet, sondern dass in vielen Fällen die Nervenendigungen an der Membrana basilaris einer wahren Atrophie anheimfallen. Die Kranken werden taub nicht nur deshalb, weil sie ein verdicktes Trommelfell, eine unbewegliche Gehörknöchelchenkette und einen ankylosirten Stapes besitzen, sondern es liegen dem auch Veränderungen in der Umgebung des runden Fensters zu Grunde, welche nicht ausser Acht gelassen werden dïrfen und bis jetzt leider nicht entspreehend gewürdigt worden sind.

In einer vor Kurzem erschienenen Arbeit Grunert's uber die Extraction des Stapes werden die von Politzer ${ }^{2}$ ) zuerst geschilderten Veränderungen am runden Fenster und seinen Membranen deutlich beschrieben und gezeigt, dass sie es sind, die alle operativen Eingriffe illusorisch machen, sei es, dass die Operation die ganze Gehörknöchelchenkette betrifft oder den Stapes allein.

In 26 Fällen von Stapesankylose fand Grunert $17 \mathrm{ma}$ Ver-

1) Ueber die vordere Tenotomie u. s. w. Jena, Gustav Fischer 1894. S. $10-11$.

2) Comptes rendus du Congrès intern. Otolog. Bruxelles 1888. 
änderungen des runden Fensters, die auch nach der Extraction. des Stapes den Ausfluss der Labyrinthfluissigkeit sicher verhindert. hätten. Diese Veränderungen bestehen in den leichtesten Fällen in einer Verdickung und Verkalkung der Membrana tympani secundaria, es wurden aber auch Hyperostosen (Moos) und selbst gänzliche Verschliessung der Fenestra rotunda (Politzer) beobachtet.

Man wird leicht begreifen, wie schwierig es für die Functionsprüfung ist, Veränderungen dieser Theile festzustellen und das Resultat des operativen Eingriffes am Schallleitungsapparate im Voraus zu beurtheilen. Noch schwieriger gestalten sich die Verhältnisse dadureh, dass die pathologischen Veränderungen von der Gegend der beiden Fenster aus sich auch ins Innere des Labyrinths fortpflanzen und die Acusticusendigungen ergreifen ( $\mathrm{P}_{0}$ litzer). Kessel und Andere glauben, dass, solange der nervöse Apparat intact ist, das Hörvermögen auch bei erkranktem Mittelohr relativ gut sich erhält. Patienten mit ankylosirten Gehörknöchelchen können Höhe und Klangfarbe einer Stimme ganz normal percipiren, vorausgesetzt, dass die Intensität der letzteren ihrer Hörfähigkeit entspricht, und dass das Labyrinth intact ist.

Anders verhält es sich da, wo Töne von tiber 20000 Schwingungen nicht mehr percipirt werden; hier ist bereits eine qualitative Veränderung des Gehörs eingetreten, und die Consonanten werden bereits sehr schlecht gehört. Ist jedoch die Hörfähigkeit bis unterhalb der Grenze von 9- oder 10000 Schwingungen gesunken, dann nimmt die Taubheit rapid zu; werden nur mehr die drei unteren Octaven gehört, so ist dies ein Zeichen stark vorgesehrittener Hörstörung.

In jenen Fallen, wo hereditäre Degenerationsprocesse zu Grunde liegen, oder als atiologische Momente das Alter, Gicht, oder nicht näher aufgeklärte individuelle Dispositionen angenommen werden können, wird sich jeder Eingriff eher schädlich als niltzlich erweisen.

Ausserdem muss man mit Miot das Allgemeinbefinden der Patienten in Betracht ziehen und bei Allgemeinerkrankungen von jeder Operation absehen.

Die Operation muss demnach vorgenommen werden, solange das Labyrinth noch intact ist, i. e. viel früher, als es bisher getibt wurde. Bevor man an die Operation geht, muss man sich auch über die hereditären Verhältnisse, ủber den Krankheitsverlauf, den Charakter der subjectiven Geräusche nnd die Ergebnisse 
der Funetionsprtifung eingehende Rechenschaft geben; leider ist gerade die letztere nicht immer im Stande, uber den Zustand des Acusticus genauen Aufschluss zu bieten, was besonders in den Initialstadien der Erkrankung hervortritt.

Bei der atrophischen Sklerose mit ausserordentlich dunnem Trommelfell, sowie da, wo eine Hyperämie des Promontoriums ersichtlich ist, erscheint mir auf Grund eigener Erfahrungen jeder operative Eingriff als ganz nutz- und resultatlos. Uebrigens ist in Fallen dieser Art die Ernäbrung des mittleren und inneren Ohres so hochgradig herabgesetzt, dass jeder operative Eingriff fast "a blane" gemacht werden kann, d. h. es findet während und nach der Operation keine irgendwie erhebliche Blutung statt.

Die ubrigen Indicationen wurden von der Mehrzahl derjenigen Otologen, welche sich mit der Frage der chirurgisehen Behandlung dieser Fälle abgegeben haben, genau abgegrenzt. Die Perception für die Uhr vom Knochen aus muss intact sein und darf keine Verktirzung aufweisen. Die C- und A-Stimmgabeln müssen auf der erkrankten Seite besser gehört werden, wie auf der gesunden.

Nur in den Fällen, wo beide Ohren gleichmässig erkrankt sind, kann die Stimmgabel vom Seheitel aus im ganzen Kopf, und ohne bestimmte Localisation auf eine Seite, gehört werden.

Ist der Vals alva'sehe Versuch, das Politzer'sehe Verfahren oder der Catheterismus der Tuba Eustachii von einer anch nur geringen Hörverbesserung gefolgt, so beweist dies, dass der Fall zur Operation geeignet ist und eine ginstige Prognose zulässt. Nach meinen eigenen Erfahrungen und den Beobachtungen, die Mounier ${ }^{b}$ beim letzten Congress der Société Française d'Otologie mittheilte, gründet sich eine der wesentlichsten Indicationsstellungen auf das Ergebniss der explorativen Myringotomie. Bei Patienten, welehe nach ktinstlicher Perforation oder partieller Trommelfellex cision eine Besserung verspüren, kann man immer auf einen mehr oder weniger günstigen Erfolg rechnen.

In einer jüngst erschienenen Arbeit hat Behrens ${ }^{2}$ ) dieselbe Meinung zum Ausdruck gebracht. Er schreibt ebenfalls der explorativen Incision des Trommelfelles einen grossen Werth in diagnostischer und prognostischer Beziehung zu. In den Fällen,

1) Bulletins et Mémoires de la Société Française d’Otologie. Mai 1897.

2) Removal of the drumhead and malleus in cases of negativ Rinne. International medical Magazine. 1897. No. 4. 
Ueber die chirurgische Behandlung der Otitis media chronica sicca. 191

wo diese Incision keine Besserung zur Folge hat, soll man nach seiner Angabe auf die operative Behandlung verzichten.

Garnault3) spricht im Gegensatz dazu der Probeincision jede prognostische Bedeutung ab, insofern ihr Erfolg ein negativer ist. Er citirt bei dieser Gelegenheit die Krankengesehichte einer Patientin, Frl. A. J., Schauspielerin. In diesem Falle war jedoch, was bemerkt werden muss, im Trommelfell eine grosse vernarbte Perforation nach einer Otorrhoe vorhanden. Die Operation hatte eine Hörverbesserung zur Folge.

Unsere eigene Erfahrung gestattet uns die Bestätigung der Resultate der meisten übrigen Collegen. In allen Fällen, wo die Paracentese allein noch keine Verbesserung brachte, war auch die weitere Operation ohne Resultat geblieben. Ich betrachte daher die explorative Incision des Trommelfelles als eine wesentliche Ergänzung der durch die Funetionsprüung gewonnenen Resultate, und als einen wichtigen Behelf bei der Indicationsstellung der chirurgischen Behandlung.

Operationstechnik.

Die früher beliebten kleineren Eingriffe, wie die Tenotomie des M. tensor tymp. und die Mobilisation des Stapes, welche eine seinerzeit gern getibte Operation war, sollen hier nur erwähnt werden, da ihre Resultate, unserer Ansicht nach, es niemals verdient haben, ernst genommen zu werden. Sohon auf dem Pariser Congress 1889 habe ich mich gegen die Stapesmobilisation in Fällen von Otitis media chronica sicea gewendet. Ich habe namentlich hervorgehoben, dass Sehwierigkeiten verschiedenster Art diese Operation zur Zeit in ibren Erfolgen illusoriseh gestalten, sowie dass der entzlindliche Reactionsprocess neue Synechien schaffen und so den ursprtinglichen Zustand noch verschlechtern kann.

Grunert hatte in seiner Arbeit tiber die Stapesextraction den grössten Theil meiner Schlussfolgerungen bestätigt, indem er eine durch diesen Eingriff erzielte Besserung als höchst nnwahrscheinlich erklärte und folgenden Satz aus der Inaugural-Dissertation von Straaten (Halle a. S. 1895) citirte: "Die Mobilisation wird nur den chronischen entzündlichen Process in einen acuten verwandeln; die momentane Verbesserung wird sehr bald einer definitiven Verschlimmerung weichen, da die Mobilisation

1) Traitement chirurgical de la surdité et des bourdonnements. Paris 1897. p. 139 etc. 
nur ein Reiz ist, welcher den Fortschritt der Krankheit beschleunigt. Aus diesem Grunde ist auch das reservirte Verhalten der deutschen Otologen der Stapesmobilisation gegentiber gerechtfertigt".

Nur die mit der Abtragung des Hammergriffes verbundene Myringodektomie hat mehrere kiberzeugte Anhänger gefunden, darunter auch Miot, der am vorigen Congress der Société Française d'Otologie diese Methode empfahl, weil er es 1. als uberflüssig betrachtet, den tympanalen Knochenring, wie Luca e vorschlägt, abzutragen, da die Perforation ohnedies permanent offen bleibt, und weil er 2. so viel wie möglich von der Kette den Gehörknöchelehen erhalten will, da jedenfalls die Gehörsehärfe der Intactheit derselben proportional ist. Letzteres wird, nach Miot, durch klinische Befunde bestätigt. Kranke, denen von den Gehörknöchelohen niohts als der - gut bewegliche Stapes geblieben ist, können wohl die Uhr in einer Entfernung von 20-30 Cm. noch hören, sie sind aber nicht im Stande, auf dieselbe Entfernung an einer allgemeinen Conversation theilzunehmen.

Ueberdies glaubt Miot, dass bei Operirten, deren Knöchelchenkette selbst keine auffallenden pathologischen Veränderungen aufweist, durch die Erhaltung des Processus brevis und des peripheren narbigen Ringes die Anlegung eines künstlichen Trommelfelles erleichtert wird. Nur in Fällen, wo die Kette ganz starr und rigid ist, empfiehlt Mi ot deren vollständige Entfernung, um jedes Hinderniss fur die Schallleitung za beseitigen. Behrens (l, c.) empfiehlt dieselbe Methode.

Extraction der Gehörknöchelchen.

Da die Stapesextraction sich als unzureichend erwies, haben einige kuhnere Otologen die Extraction der ganzen Knochenkette vorgeschlagen, deren Starrheit sie als eine der Hauptursachen der Schwerhörigkeit betrachteten.

So war man von dem Versuche einer theilweisen Entfernung des Trommelfelles zu einer vollständigen Extraction der Gehörknöchelchen vorgeschritten.

Samuel Sexton ${ }^{1}$ ) in New-York erklärte sich ebenfalls als Anbänger dieser Methode und publicirte eine grössere Anzahl von Fällen, in denen er mit ihr Erfolge erzielte. Trotzdem konnten

1) System of Diseases of the Ear, Nose and Throat 1894, und Arch. of Otol. Vol. XX. No. 2. p. 95 . 
sich noch einige Jahre hindurch die meisten Otologen nicht entsohliessen, diese Operation in die Praxis einzufuhren. Sie wurde seitdem in den meisten Ländern versucht, und das mit verschiedenen Resultaten.

Garnault hat in Frankreich diese Operation mit vielem Eifer vertheidigt; aber seine Schlussfolgerungen kranken an dem Fehler, dass er zwischen den post-otorrhoeischen Synechien und der Otitis chronica sicea keinen Unterschied macht. Analysirt man seine Fälle, so zeigt es sich, dass eine Besserung nur bei solchen der ersterwähnten Categorie zu constatiren ist, während bei der Otitis chronica sicea, der diese Zeilen gewidmet sind, Erfolge nicht zu verzeichnen waren. Es ist sehr bedauerlich, dass der Verfasser diesbezüglich nicht jene scharfe Grenze gezogen hat, wie sie Politzer ${ }^{1}$ ) bereits im Jahre 1884 auf dem Congresse in Basel formulirt hat.

Eine lange Reihe von Jahren hindurch wurde die Extraction der Knöchelchen (ansgenommen den Stapes) durch den äusseren Gehörgang vorgenommen, und Sexton hat zu diesem Zwecke eine Reihe von Instrumenten angegeben, welche die Ausfuhrung bedeutend erleichterten. Als Stack e im Jahre 1890 zu Berlin seinen bekannten Vortrag über die Behandlung der Mittelohreiterungen und die Extraction der Gehörknöchelchen mittels der Eröffnung der Trommelhöhle von der Regio retroauricularis aus hielt, wurde auch von mehreren Chirurgen vorgeschlagen, bei der sklerosirenden Otitis das Cavum tympani auf demselben Wege $z u$ eröffnen.

In Frankreich wurde diese Methode besonders von Garnault empfohlen. Es sind demnach 2 Methoden zur Verfügung, um die Gehörknöchelchen zu entfernen: Die eine, einfache und leichte, erreicht das Operationsgebiet durch den äusseren Gehörgang, die zweite, schwierigere und relativ gefährlichere, sucht es vom Warzenfortsatz aus zu erreichen.

Wir halten es für überflüssig, diese Methoden selbst näher zu beschreiben, da sie jedem Otologen genügend bekannt sind. Hier handelt es sich vielmehr um die Frage, ob die Eröffnung vom Warzenfortsatz aus, welche einen bedenklichen und folgenschweren Eingriff darstellt und eine längere und complicirte Nachbehandlung erfordert, auch Vortheile aufzuweisen hat, um

1) Operative Behandlung der Hörstörungen nach abgelaufener Mittelohreiterung. Congrès periodique internat. d'Otol. Bâle 1884. p. 296. 
diese Nachtheile zu paralysiren. Garnault hebt in seiner Arbeit als besonderen Vortheil die Leichtigkeit hervor, mit welcher man die Knöchelehen abtragen und den Stapes zu Gesicht bekommen kann. Dadureh kommt man in die Lage, auch an der Peripherie des ovalen Fensters incidiren und so dem Knöehelchen diejenige Beweglichkeit ertheilen zu können, deren es fttr seine normale Function bedarf. Es gelingt indess nicht immer so leioht, die Basis des Steigbügels zu umschneiden, weil der Knochenwall des Fensters oft so stark vorspringt, dass der obere Theil der Platte dadurch verdeckt wird. Andererseits kann, meiner Ansicht nach, wie Garnault ) auch zugiebt, dureh den operativen Eingriff eine Hyperämie, ja Hämorrhagie im Labyrinthe erfolgen, welche die Knochenperception aufheben, transitorischen Schwindel erzeugen und $\mathrm{zu}$ consecutiver Taubheit führen kann.

Garnault selbst sagt: "Wenn ich auch bei einigen Patienten durch die chirurgische Behandlung eine Verbesserung des Gehörs erzielt habe, so sind doch auch andere da, bei denen dieselbe Operation einen entziindlichen Process im Centralapparat hervorgerufen hat, der die Erregungsfähigkeit des Nerven herabsetzte."

In einigen Fällen wurde transitorisch eine Herabsetzung der Knochenperception für die Uhr constatirt, welche nach einiger Zeit wieder schwand. Immerhin giebt es aber auch noch Fälle, wo diese Perceptionsfähigkeit nicht wieder zurtickkehrt. Ich erinnere mich des Patienten, den Garnault in seiner letzten Arbeit erwähnt, und der in meiner Gegenwart operirt wurde. Bei diesem wurde die Uhr in der Frontal- and Occipitalregion gar nicht, und vor dem Gehörgang und am Proc. mastoid. nur sehr verkürzt vom Knochen aus pereipirt.

Diese Nachtheile allein mitissen schon den Operateur sehr vorsichtig machen, auch wenn er das ganze Operationsgebiet deutlich zu ubersehen vermag. Aber ausserdem ist noch eine ganze Reihe anderer iibler Zufälle mit dem Stacke'schen Operationsverfahren in Zusammenhang. Auf der einen Seite besteht die schwierige Abhaltung der Infection von der Wunde, solange sie nicht vollständig überhäutet ist, auf der anderen die Bildung narbiger Synechien, die den gesetzten Knochendefect vollständig ausfüllen und auch den Steigbügel wieder verdecken.

1) Traitement chirurgical de la surdité et des bourdonnements. Paris 189i. p. 139. 
Ueber die chirurgische Behandlung der Otitis media chronica sicca. 195

Kurz zusammengefasst, miissen wir demnach sagen, dass das äussere oder retroauriculäre Operationsverfahren wohl eine viel bessere Uebersicht der Gegend des ovalen Fensters und eine leiehtere Zugänglichkeit dieser Theile für verschiedene Eingriffe ermöglicht, dass aber folgende Punkte zu beachten sind.

1. Secundäre Infection der Operationswunde. Eine sehr häufige, den Erfolg der Operation in Frage stellende Complication. Die dauernde Secretion fördert die Bildung der Granulationen, welche verhindert werden soll. Gleichzeitig besteht, wie bei jeder Otorrhoe, eine permanente Gefahr für den Patienten, da stets neue und lebensgefährliche Complicationen hinzutreten können.

2. In den günstigen Fällen, wo die Heilung ohne Eiterung erfolgt, kann noch immer durch Bildung von Pseudomembranen und narbigen Synechien eine Verengerung des Gehörganges Platz greifen, so dass es eventuell unmöglich wird, den Stapes wieder zu erreichen, da er verborgen und in Narbengewebe eingebettet ist. Der Patient wird nunmehr unheilbar taub, da einerseits das Leiden fortschreitet, andererseits die Behandlung ihr Resultat völlig verfehlt.

Operirt man hingegen vom äusseren Gehörgang aus, so gelingt es in den meisten Fällen bei einiger Erfahrung und Uebung, in wenigen Minuten das ganze Trommelfell sowie Hammer und Amboss zu entfernen, ohne eine der oben erwähnten Complicationen befiurehten zu muissen.

Es wird sogar durch die Extraction der beiden genannten Knöchelohen selbst bereits eine derartige Erschütterung in der ganzen Kette bewirkt, dass der Steigbtigel gleichzeitig von selbst mobilisirt wird.

Beabsichtigt man jedoch, den Steigbügel direct zu mobilisiren, so kann - in Chloroformnarkose - vom Gehörgang aus die knöcherne Umwandung, welche den Stapes maskirt, weggemeisselt und dann, nach Extraction von Hammer und Amboss, die Mobilisirung des Steigbügels ausgeführt werden. Bei den von mir operirten Fällen erzielte ich sehr gute Erfolge durch die Entfernung der zwei ersten Knöchelchen allein, ohne weitere directe Mobilisation des Stapes.

Ioh brauche nicht hinzuzufügen, dass diese Operation keine weiteren Complicationen nach sich zieht, die Vernarbung ist nach Verlauf eines Monats vollendet, es kommt zu keiner secundären Infection, und der Patient kann bald wieder seiner Beschäftigung nachgehen. Allerdings muss zugegeben werden, dass während 
des Heilungsprocesses sich oft Pseudomembranen bilden, welche, an Stelle des entfernten Trommelfelles, die Oeffnung verschliessen und einen neuerlichen Eingriff erfordern. In einem solchen Falle war ich sogar genöthigt, zweimal derartige narbige Membranen abzutragen. In den meisten Fallen geht jedoch die Heilung normal vor sich, es bildet sich ein theilweiser, membranöser Verschluss am Margo tympanicus ans, der jedoch eine gentigende Oeffnung lässt, um die Paukenhöhle überblicken zu können.

Es bandelt sich somit um eine höchst einfache Operation auch da, wo man den Steigbügel direct mobilisirt oder den thn umrandenden Knochenwall abmeisselt.

Vergleich der beiden Methoden in Bezug anf ihre Resultate.

Da ich tuber die Erfolge des Stacke'schen Verfabrens keine Erfahrungen besitze, werde ich die von Garnault in seinen Fällen erzielten Resultate behufs Vergleichung mit den von mir dureh den Gehörgang vorgenommenen Operationen heranziehen.

Garnault hat mir Fräulein von H, von der in seiner Arbeit die Rede ist, vorgestellt. Diese Patientin litt an einer Sklerose und hörte offenbar nach der Operation besser als vor derselben, aber immerhin ist ihre Hörschärfe nicht sehr beträchtlich, da sie, wie ich in Gemeinsehaft mit Collegen Miot feststellte, auf dem besseren Ohr Flüstersprache nur auf $20-25 \mathrm{Cm}$, Conversationsspracbe auf $60-80 \mathrm{Cm}$. Maximum hörte.

Ein zweiter Patient, Herr Rondot, 69 Jahre alt, erfuhr ebenfalls eine Verbesserung seines Gehöres, aber er hört doch die Conversationssprache nur auf $40-50 \mathrm{Cm}$., und zusammen mit Miot konnte ich constatiren, dass ein fibröses Diaphragma die ganze Paukenhöhle absehloss, so dass Garnault zur Anwendung des Galvanokauters genöthigt war.

Ich sehe dabei ganz ab von einigen Fällen, bei welchen durch die Stacke'sche Methode gar kein Erfolg erzielt wurde, da es ja bei jedem Verfahren Fälle giebt, wo es vollständig ver sagt. Aber ziehen wir auch nur die ginstigen Erfolge in Betracht, so müssen wir sagen, dass man nach Stacke keine besseren Resultate erzielt, als beim Operiren vom äusseren Gehörgang aus. Ioh habe unter meinen Fällen solche von Hammer-Ambossextraction, die jetzt, 18 Monate nach der Operation, ebensogut hören wie unmittelbar nach dem Eingriff, allerdings anch nicht besser wie die von Garnault. 
Ich erinnere mich unter anderen eines 30 jährigen Fräuleins, das ich 12-15 Jahre lang mittelst Catheterismus, Massage und aller anderen gebräuchlichen Mittel behandelte. Während die Patientin früher laute Stimme nur ad concham hörte, vernahm sie, unmittelbar nach der im Mai 1896 vorgenommenen Operation (Abtragung von Trommelfell, Hammer und Amboss) auf eine Entfernung von 50-60 Cm. anf der operirten Seite. Es bildete sich bei dieser Patientin weiterhin eine der erwähnten Pseudomembranen aus, und der ganze Operationseffect schien verloren. Nach einem nochmaligen Eingriff, bei welchem dieses Hinderniss beseitigt wurde, blieb die Perforation offen, die Paukenhöhle ist seither ganz trocken, und die Hörschärfe ziemlich unverändert, solange die Patientin nicht an Schnupfen leidet, der sie etwas zu beeinträchtigen scheint.

Bei einer zweiten Patientin war die Verbesserung noch beträchtlicher. Nach einer einfachen Myringotomie stieg bier die Hörweite von $10 \mathrm{Cm}$. auf $50-60 \mathrm{Cm}$. für Conversationssprache, und nach Abtragung von Hammer und Amboss ergab sich ein weiterer Zuwachs von 25-30 $\mathrm{Cm}$, welcher Zustand seither unverändert anhält. Diese gleichmässig günstigen Resultate zeigen, dass die Operation vom Meatus externus aus der anderen wegen ihrer Einfachheit, schnellen Ausführbarkeit und völligen Complicationslosigkeit vorzuziehen ist. Bisher habe ich nur meine eigenen, persönlichen Erfahrungen vorgebracht. Ioh weiss aber, dass meine Collegen Miot und Mounier aus Paris bei Einschlagung desselben Verfahrens ebenfalls Besserungen erreichten. Dennoch betrachte ich die Frage als noch unentschieden und glaube vielmehr, dass nooh weitere Beiträge nöthig sind, um zu einer definitiven Anschauung uber den relativen Werth beider Methoden zu gelangen.

Selbstverständlich wird die Stacke'sche Operation, wenn sie sich als die erfolgreichere erweisen sollte, trotz aller ibrer Nachtheile die Operation der Zukunft bei der Otitis chronica sicea sein. Falls aber die Endergebnisse beider Methoden sich als annährend gleichwerthig darstellen sollten, so wird die andere vorzuziehen sein, als einfacher und ungefährlicher.

Künstliches Trommelfell.

Bei beiden Verfahren ist der unmittelbare Erfolg oft kein auffälliger. In manchen Fällen ist sogar die Hörschärfe nach der Operation geringer als vor derselben. In solchen Fällen kann 
man die Anwendung eines künstlichen Trommelfelles versuchen, welches aus einem Wattekẗgelchen besteht, das mit Vaselin oder Carbolglycerin befeuchtet und in die Trommelhöhle eingeführt wird. Es ist nicht nöthig, dieses künstliche Trommelfell direct gegen den Stapes zu dirigiren. Ich habe sogar mehrere Patienten gesehen, z. B. den von Garnault operirten Herrn G., bei dem das ktinstliche Trommelfell bedeutend besser wirkte, wenn es in den hinteren unteren Theil der Paukenhöhle eingeführt wurde, als wenn man es in der Stapesgegend anlegte. Die beste Wirkung ergab sich bei Anlegung in der Region des runden Fensters. Es ist ausserdem von Wichtigkeit, das küstliche Trommelfell mit einem Fette zu imprägniren, um eine seröse Exsudation der Paukenschleimhaut hervorzurufen, welcher Umstand eine bessere Haftung an der Paukenhöhlenwand und damit erhöhte Wirksamkeit des klinstlichen Trommelfelles mit sich bringt. Jedoch mulssen die betreffenden Patienten diesbezüglich instruirt und ihnen grösste Vorsioht anempfohlen werden, um eine eiterige Transformation des serösen Exsudates zu vermeiden.

\section{Extraction des Steigbügels.}

Wir werden nur in aller Kulrze auf diese Operation eingehen, welche sich bei der Behandlung der Otitis chronica sicca als fast völlig wirkungslos erwiesen hat. Zuerst wurde sie 1877 von Kessel ansgeführt. Es handelte sich um ein schwerhöriges Mädchen, welches auch an subjectiven Geräuschen litt. Das Resultat war blos eine Verminderung der subjectiven Erseheinungen; die Taubheit blieb unverändert.

Dieselbe Operation wurde seither mit gleich negativem Erfolge von Bezold, Blacke, Botery, Jack u. A. ansgefthrt. Neuerlich beschäftigte sich $\mathrm{Gr}$ une $\mathrm{r} t$ eingehend mit dieser Frage und theilte drei so behandelte Fälle von Otitis media chronica sicea aus der Ohrenklinik in Halle a. S. mit.

Im ersten Fall blieben Schwerhörigkeit und subjective Ersebeinungen nach Extraction des Stapes und Eröffnung des Vorhofes ganz unverändert, jedoch zeigte sich nach derselben eine leichte Facialisparese.

Im zweiten Fall war der Stapes von einer Knochenmasse umgeben, die das ovale Fenster vollständig ausfüllte. Operation ohne Resultat.

Im dritten Fall konnte der Stapes nicht aufgefunden werden, da er ohne Zweifel mit dem Tampon entfernt worden. Der 
Ueber die chirurgische Behandlung der Otitis media chronica sicca. 199

Patient litt noch einige Wochen hindurch an Schwindel. Sonst derselbe Zustand wie vor der Operation.

Man sieht, dass es mit dieser Operation bisher noch nicht gelungen ist, irgend eine Besserung zu erzielen. Es wurden vielmehr - im Gegentheil - von Deneh ${ }^{\text {) }}$ Fälle beobachtet, wo nach derselben mehrere Monate lang Schwindelanfälle, ja selbst Vermehrung der Schwerhörigkeit sich einstellten. Nur J a ck ${ }^{2}$ ) hat diese Operation mit einem gewissen Eifer verfochten. Da aber seine Resultate mit denen anderer Autoren nicht übereinstimmen, so glaubt Grunert, dass der Optimismus dieses Forsohers ihn $\mathrm{zu}$ weit führt.

In einer gründlichen Arbeit aber die operative Behandlung der Otitis media sicca reproducirt $\mathrm{Ch}$ e atl $\mathrm{e}^{3}$ ) die Ansichten fast der meisten Otologen der Welt, welche er entweder direct als Antwort auf seine Anfragen bekommen oder ihren Publicationen entnommen hatte. Leser, welche sich für diese Frage besonders interessiren, seien daher hier auf dieses Werk verwiesen.

\section{Sehlussfolgerungen.}

1. In Fällen von Otitis chronica media sicca ist man, wenn die gewöhnlichen Behandlungsmethoden (Lufteintreibungen, directe und indirecte Massage etc.) wirkungslos bleiben, autorisirt, bei intactem nervösen Apparat die chirurgische Behandlung einzuleiten.

2. Die explorative Paracentese des Trommelfelles ist ein sehr werthvolles diagnostisehes und prognostisches Mittel; $\mathrm{Pa}$ tienten, die danach keine Besserung empfinden, durfen nicht operirt werden, weil hier ein Erfolg ausgeschlossen ist.

3. Die Operation soll vorztiglich vom äusseren Gehörgang aus ausgefubrt werden. Sie besteht in der Abtragung des Trommelfelles und der Gehörknöchelchen mit Ausnahme des Stapes. In jenen wenigen Fällen, wo die localen anatomisohen Verhältnisse die leichte Entfernung des Amboss und die Siohtbarkeit des Stapes beeinträchtigen, soll man den oberen hinteren Theil des Margo tympanicus wegmeisseln. Auch dieser letztere Eingriff soll vom Meatus externus aus vorgenommen werden.

4. Während der Abtragung des Trommelfelles und der

1) New-York Medic. Journal v. 26. Sept. 1897.

2) Transactions of the American Otological Society 1893, u. Boston Medical Surgical Journal. 10. Jan. 1895.

3) The Practitioner. Mai 1897. p, 494. 
200 X. MOURE, Ueber die chir. Behandl. der Otitis media chronica sicca.

Knöchelchen wird die ganze Kette in eine genügende Erschütterung versetzt, um den Stapes von selbst and ohne weiteren directen Eingriff zu mobilisiren.

5. Die retroauriculäre Operation hat sich bisher nicht als erfolgreicher erwiesen als die durch den Gehörgang. Sie besitzt vielmehr grosse Nachtheile, da sie active Congestionszustănde, ja auch vestibuläre Blutungen hervorrufen kann. Auch eine Infection von aussen mit consecutiver Eiterung in den Mittelohrräumen ist nicht ausgeschlossen. Die Operationsöffnung wird weiterhin auch sehr bald von bindegewebigen Synechien erfullt, welche die ganze Paukenhöhle auskleiden. Die bisher durch dies genannte Operationsverfahren erzielten Resultate sind nicht geeignet, diese Anschauungen zu widerlegen.

6. Die operative Behandlung hat nicht immer gleichzeitig Verbesserung des Gehörs und Beeinflussung der subjectiven Symptome zur Folge. Oft geschieht es, dass, bei einer Besserung der Hörfähigkeit, die subjectiven Geräusche unverändert bleiben, und umgekehrt.

7. Die bisherigen Erfahrungen lassen noch kein bestimmtes Urtheil über die voraussichtliche Dauer der eingetretenen Besserung zu. Wir sind deshalb heute noch nicht in der Lage zu entscheiden, ob die Resultate nicht vielleicht blos voribergehende sind.

8. Wurde durch die Abtragung des Trommelfelles und der Gehörknöchelchen kein Erfolg erzielt, so ist die Anlegung eines klinstlichen Trommelfelles zu versuchen. Dieses muss nicht immer in der Gegend des Stapes plaeirt werden, man kann es vielmehr auch in dem hinteren unteren Theil der Paukenhöhle, entsprechend der Gegend des runden Fensters, anbringen. Es besteht aus einem mit Vaselin oder Carbolglycerin getränkten Wattekügelchen. Seine Anwesenheit in der Paukenhöhle ruft häufig eine seröse Exsudation hervor, die streng controlirt werden muss, damit ihr Uebergang in eine eiterige verhutet werde. 